\title{
13. Gesundheitspflege-Kongress
}

— Am 9. und 10. Oktober 2015 findet im Radisson Blu Hotel in Hamburg der 13. GesundheitspflegeKongress von Springer Medizin statt. Erwartet werden 800 Kongressteilnehmerlnnen - Pflegefachkräfte, Stations- und Pflegedienstleitungen, PflegedirektorInnen, Lehrende und Studenten. Eröffnet wird der Kongress von Cornelia PrüferStorcks, Senatorin für Gesundheit und Verbraucherschutz, Hamburg. Der Kongress bietet ein hochaktuelles Fortbildungsprogramm, das Springer Medizin in bewährter Weise mit den Kooperationspartnern des Kongresses - dem Albertinen-

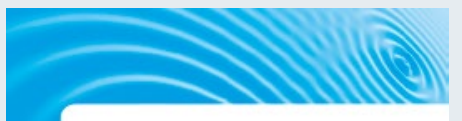

13. GesundheitspflegeKongress Hamburg | 9.-10.10.2015

\section{SpringerMedizin}

dem Deutschen Berufsverband für Pflegeberufe (DBfK), der Hochschule für Angewandte Wissenschaften Hamburg sowie den Universitätskliniken Hamburg-Eppendorf und Schleswig-Holstein - zusammen gestellt hat. Arbeitsgestaltung und Personalsituation in den Kliniken, Entlastung der Pflegekräfte durch neue Aufgabenverteilung, Einsatz akademisierter Pflegekräfte und ausländischer Fachkräfte, das Neue Pflegeberufegesetz stehen ebenso auf dem Programm wie Demenz- und Hygienemanagement und viele weitere Fortbildungsthemen. Ein PflegeKolleg live zur Kompressionstherapie, ein Science Slam, bei dem Projekte aus der Pflegewissenschaft unterhaltsam präsentiert werden und eine Gesprächsrunde für pflegende Angehörige ergänzen das Angebot.

www.gesundheitskongresse.de

bpa

\section{Mangel an Heimplätzen}

— "Bereits in vier Jahren werden Heimplätze in bislang ungeahntem Ausmaß fehlen", warnt Stefan Kraft, Beauftragter der Landesgruppe Baden-Württemberg des Bundesverbandes privater Anbieter sozialer Dienste (bpa). „Neben einem überproportional wachsenden Bedarf an Heimplätzen droht paradoxerweise der größte Platzabbau in der Geschichte des Landes", so Kraft. Ursache ist dem bpa zufolge die Landesheimbauverordnung, die von den Bestandseinrichtungen ab 2019 umgesetzt werden soll. Sie sieht neben dem Einzelzimmergebot eine Reihe von Anforderungen an die Größe und die Breite von Zimmern, Wohngruppengröße u.a. vor, durch die ein massiver Platzabbau drohe. Allein durch das Doppelzimmerverbot könnten rund 17.000 Plätze wegfallen. Der bpa fordert, die allgemeine Übergangsfrist der Verordnung deutlich zu verlängern und - wo möglich umfassende Befreiungen bestehender Einrichtungen durch die Heimaufsicht.

www.bpa.de

\section{Förderprogramm \\ Menschen mit Demenz in der Notaufnahme}

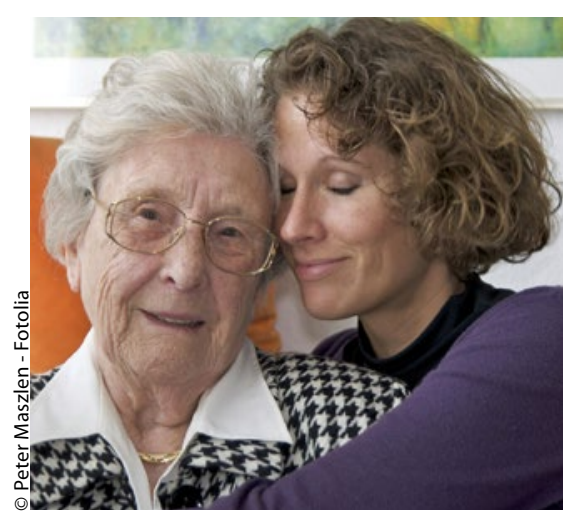

— In Deutschland werden $70 \%$ der Pflegebedürftigen zuhause betreut. Welche Folgen die hohe emotionale und psychische Belastung für pflegende Angehörige haben kann, hat jetzt eine Studie des Meinungsforschungsinstituts Forsa für die Private Pflegeberatung Compass untersucht. Die Ergebnisse sind alarmierend. Jeder Zweite
(65\%) fühlt sich mit der Pflege von Angehörigen manchmal bis häufig überfordert. Und 73\% der Pflegenden empfinden die Tätigkeit emotional und psychisch eher bis sehr belastend. Jeder Dritte (32\%) gab außerdem an, dass die Belastung vorübergehend zu depressiven Phasen geführt habe. Bei Menschen, die demenzkranke Angehörige betreuen, steigt dieser Anteil sogar auf $40 \%$.

Umso wichtiger sei es, Entlastungsangebote anzunehmen, um sich zu erholen und Kraft zu tanken, betont die Private Pflegeberatung. Die Kosten hierfür würden in vielen Fällen von der Pflegeversicherung übernommen. Allerdings hat die ForsaUmfrage diesbezüglich ein Defizit aufgedeckt: Jeder zweite Befragte (50\%) fühlte sich schlecht oder weniger gut über bestehende Entlastungsangebote informiert.

www.forsa.de
— Die Robert Bosch Stiftung fördert seit Juli für drei Jahre die Alexianer St. Hedwig Kliniken Berlin GmbH im Rahmen des Förderprogramms "Menschen mit Demenz im Akutkrankenhaus“ . Ziel ist, die komplexen Abläufe der Notaufnahme auf die spezifischen Bedürfnisse von Demenzerkrankten abzustimmen. Geplant sind Schulungsmaßnahmen für Mitarbeiter aller Berufsgruppen in der interdisziplinären Notaufnahme (INA). Darauf aufbauend werden Fortbildungsmodule in Zusammenarbeit mit der Alzheimer Gesellschaft Berlin entwickelt und angeboten, die räumlichen Gegebenheiten der INA angepasst. Nach erfolgter Evaluation aller Maßnahmen wird ein Manuel erstellt, das für Notaufnahmen in Akutkrankenhäusern zur freien Verfügung stehen wird.

www.alexianer-berlin-hedwigkliniken.de 